\title{
Electrical fixing of 1000 angle-multiplexed holograms in SBN:75
}

\author{
Jian Ma, Tallis Chang, John Hong, and Ratnakar Neurgaonkar \\ Rockwell Science Center, A25A, 1049 Camino Dos Rios, Thousand Oaks, California 91360
}

George Barbastathis and Demetri Psaltis

Department of Electrical Engineering, 116-81, California Institute of Technology, Pasadena, California 91125

Received April 4, 1997

We have demonstrated electrical fixing of 1000 angle-multiplexed holograms in a $1-\mathrm{cm}^{3}$ volume Ce-doped $\mathrm{SBN}: 75$ crystal. A revealing procedure yielded an average diffraction efficiency of $0.005 \%$ for each hologram, with approximately $20 \%$ variation. The erasure resistance of the fixed gratings was verified. C 1997 Optical Society of America

To achieve nonvolatile storage in holographic memory systems that use photorefractive crystals, a means for transforming optically erasable space charge gratings into optically inactive material perturbations must be developed. Nondestructive readout of photorefractive holograms can be achieved by one of two prominent methods: (1) by forming an optically inactive ionic charge grating that compensates for the volatile electronic charge grating by temperature cycling to control the ionic mobility ${ }^{1}$ or (2) by forming a ferroelectric domain grating that follows the electronic charge grating pattern by the application of an external electric field opposite in sign to a poling field at room temperature ${ }^{2-4}$ or by temperature cycling through the ferroelectric phase transition. ${ }^{5}$ Although multiplexed nonvolatile storage of large numbers of holograms in $\mathrm{LiNbO}_{3}$ has been performed ${ }^{1,6}$ by the thermal fixing method, the electrical method has not yet been successfully used to demonstrate nonvolatile storage of large numbers of multiplexed holograms. In this Letter we report the electrical fixing of 1000 holograms that are angularly multiplexed in a common recording volume of Ce-doped $\mathrm{Sr}_{0.75} \mathrm{Ba}_{0.25} \mathrm{Nb}_{2} \mathrm{O}_{6}$, strontium barium niobate (Ce:SBN75).

To record multiple holograms, either a single-cycle or a multiple-cycle approach can be used. In the singlecycle procedure, first all holograms are recorded as photorefractive gratings and then a single fixing pulse converts the composite set of holograms into a spatially varying domain pattern in one step. The multiplecycle procedure, in contrast, breaks up the sequence of exposures into several sets of smaller subsequences of exposures, with the separate revealing and fixing pulses applied after each subsequence. A revealing electrical pulse is applied before the application of a fixing electrical pulse because the crystal was depoled by the fixing pulse applied at the end of each subsequence; a revealing process is necessary to repole the crystal uniformly so that the optimum fixing condition is recovered. We found that the diffraction efficiency of fixed multiplexed holograms is much higher with the multiple cycle, primarily for two reasons. First, the holograms recorded in earlier exposure cycles have better resistance to erasure by successive exposures when the fixing pulse is applied, in comparison with the single-cycle case in which each hologram is subjected to photorefractive erasure by all subsequent exposures before the single fixing pulse is applied. Second, empirical observations indicate that multiplexed holograms of larger diffraction efficiency can be recorded when a depoling electric field pulse such as a fixing pulse is applied before recording. ${ }^{7,8}$ For these reasons we chose the multiple-cycle method to perform the 1000 fixed hologram storage experiment.

The experimental system that we used is shown schematically in Fig. 1; a doubled Nd:YAG laser provides a 532-nm source for the experiment. The laser beam is expanded before being split into reference and object beams. The reference beam is reflected off a mirror whose vertical orientation is controlled with a motorized rotation stage (Motorized Rot. Stage). The mirror is then imaged onto the crystal by a telecentric system. The crystal is placed upon a motorized stage to permit rotations in the horizontal plane. The object beam fills the aperture of a $640 \times 480$ spatial light modulator (SLM, a liquid-crystal display) before being focused onto the SBN crystal. The crystal used was Ce-doped (0.02\%) SBN:75, a cube with a volume of approximately $1 \mathrm{~cm}^{3}$. The two writing beams entered the $a$ face of the crystal at a nominal interbeam angle of $3^{\circ}$ with the grating vector parallel to

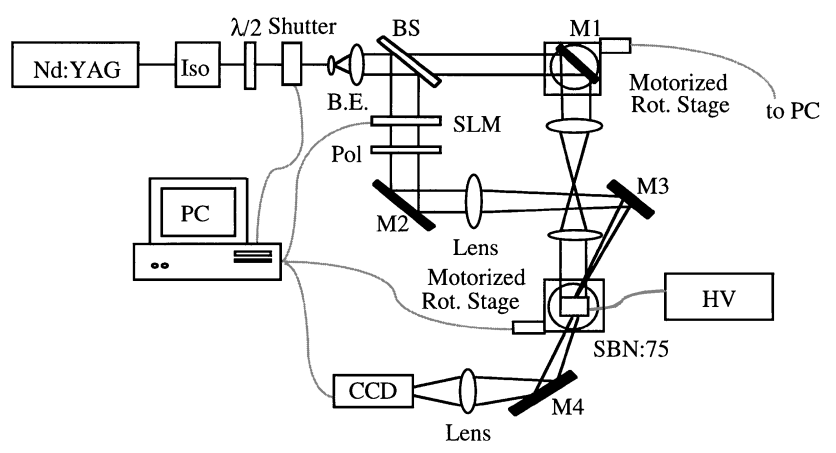

Fig. 1. Experimental setup for recording and fixing anglemultiplexed holograms: M's, mirrors; Iso, isolator; B.E., beam expander; BS, beam splitter; Pol, polarizer; PC, personal computer. 
the $c$ axis, which provides a horizontal angular selectivity of $\sim 0.15^{\circ}$ (measured by rotating the crystal until cross talk between adjacent holograms negligible). We achieved angle multiplexing by rotating the crystal in the horizontal plane $\left(0.15^{\circ}\right.$ separation $)$. Fractal (off-plane) multiplexing ${ }^{9}$ was achieved, in the same storage volume, by rotation of mirror M1 to change the angle of incidence of the reference beam in the vertical plane ( $2^{\circ}$ separation). Two hundred holograms were multiplexed on every fractal row, and five fractal rows were used, for a total of one thousand holograms. A computer was used to coordinate the operation of the spatial light modulator, shutters, high-voltage pulse generator $(\mathrm{HV})$, and a frame grabber to acquire the CCD output image data.

To ensure that a sequence of hologram exposures in a photorefractive medium will result in a uniform distribution of diffraction efficiencies, a schedule of exposures (fluence per hologram) must be followed. ${ }^{8,10}$ As mentioned above, we found that depoling the crystal before the exposures significantly improved the diffraction efficiencies of a set of multiplexed holograms. For example, the depoling pulse $(-1.8 \mathrm{kV} / \mathrm{cm}$, 0.2 -s duration, nominally square shape) leads to an average 14-fold increase in the diffraction efficiencies of 100 holograms in our sample compared with the case with no depoling pulse. ${ }^{8}$ Of course, after the holograms are recorded and before the diffraction efficiency is measured, the crystal must be repoled to restore the linear electro-optic coefficients, which degrade with the randomizing influence of the depoling pulse. One key observation that affects the diffraction efficiency is the difference in optical erasure characteristics of a single grating written with and without the depoling electric field pulse. The main contribution to the enhanced diffraction efficiency of the multiplexed holograms seems to be from the increased erasure time constant that effectively increases the diffraction efficiency of the multiplexed holograms. We found that a freshly poled crystal followed a conventional simple exponential decay of $\exp \left(-t / \tau_{e}\right)$ well. However, the erasure characteristics of holograms written with the application of a depoling electric field pulse deviated significantly from the single $\tau_{e}$ exponential decay and followed a two-timeconstant behavior that can be fitted to

$$
\begin{aligned}
\eta= & r_{1}{ }^{2} \exp \left(-\frac{2 t}{\tau_{s}}\right)+r_{2}{ }^{2} \exp \left(-\frac{2 t}{\tau_{L}}\right) \\
& +2 r_{1} r_{2} \exp \left[-t\left(\frac{1}{\tau_{s}}+\frac{1}{\tau_{L}}\right)\right], \\
r_{1}+r_{2}= & 1,
\end{aligned}
$$

where $\eta$ is the normalized diffraction efficiency, $\tau_{s}$ is the short-time constant, and $\tau_{L}$ is the long-time constant. The underlying reasons for this effect are being investigated and are beyond the scope of this Letter; however, we believe that this two-time-constant behavior is related to the participation of a second photoactive species induced by the depoling electrical pulse. The negative preparation pulse depoles the crystal to a certain degree and creates a random domain structure in the crystal. The randomly structured $\mathbf{P}_{s}$, the spontaneous polarization in the material, introduces a new charge trapping defect species, which gives rise to decay of gratings at two separate rates. ${ }^{11}$ Species related to the random domain structure are associated with a longer-time constant $\tau_{L}$, whereas the intrinsic (in a fresh state) species are associated with a shorttime constant $\tau_{s}$. An optimum preparation electrical pulse (e.g., $E_{p}=-1.8 \mathrm{kV} / \mathrm{cm}$ ) produces the most random domain structure yielding the highest density of the new species.

The optical erasure curves of a single grating were then measured for a volatile photorefractive grating (not fixed) and a fixed grating. The initial diffraction efficiency was $35 \%$ for both cases. The erasure light was a non-Bragg-matched $o$-polarized beam of $532 \mathrm{~nm}$ and $100 \mathrm{~mW} / \mathrm{cm}^{2}$. The decay curves for these two cases are shown in Fig. 2, where the rapid-decay curve corresponds to the volatile case. The slow-decay curve corresponding to the fixed grating was monitored at less frequent intervals (as indicated by the open circles). The diffraction efficiency of the fixed grating was measured after the holograms were revealed with a positive revealing electrical pulse of $5 \mathrm{kV} / \mathrm{cm}$ and 0.5 -s duration. After each measurement the crystal was quickly refixed with a fixing electric field pulse of $-1.8 \mathrm{kV} / \mathrm{cm}$ and 0.2 -s duration before being exposed to the erasure beam again for continued erasure.

An exposure schedule that accounts for the two-timeconstant behavior was derived as follows: Let $A_{m}$ be the amplitude of the $m$ th hologram recorded. When the erasure of all the subsequent exposures is taken into account, $A_{m}$ is given by

$$
\begin{aligned}
A_{m}= & {\left[1-\exp \left(-\frac{t_{m}}{\tau_{w}}\right)\right]\left[r_{1} \exp \left(-\frac{S_{m+1}}{\tau_{s}}\right)\right.} \\
& \left.+r_{2} \exp \left(-\frac{S_{m+1}}{\tau_{L}}\right)\right], \\
S_{m+1}= & \sum_{i=m+1}^{M} t_{i},
\end{aligned}
$$

where $t_{m}$ is the exposure time for the $m$ th hologram, $\tau_{w}$ is the writing-time constant, and $M$ is the total

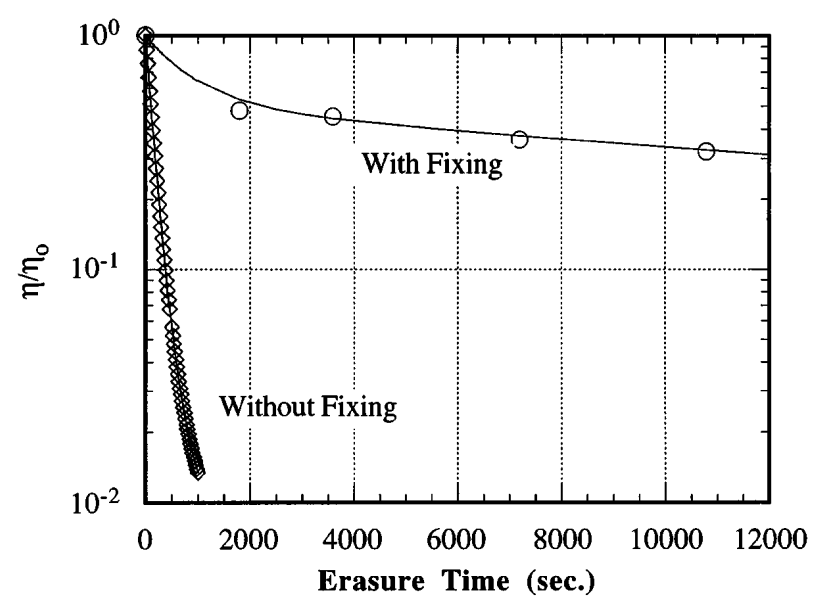

Fig. 2. Erasure of a hologram (not fixed): curve fitted with $r_{1}=0.85, r_{2}=0.15, t_{s}=250 \mathrm{~s}, t_{L}=2500 \mathrm{~s}$. Erasure of a fixed hologram: curve fitted with $r_{1}=0.293, r_{2}=$ $0.707, t_{s}=1000 \mathrm{~s}, t_{L}=50000 \mathrm{~s}$. 

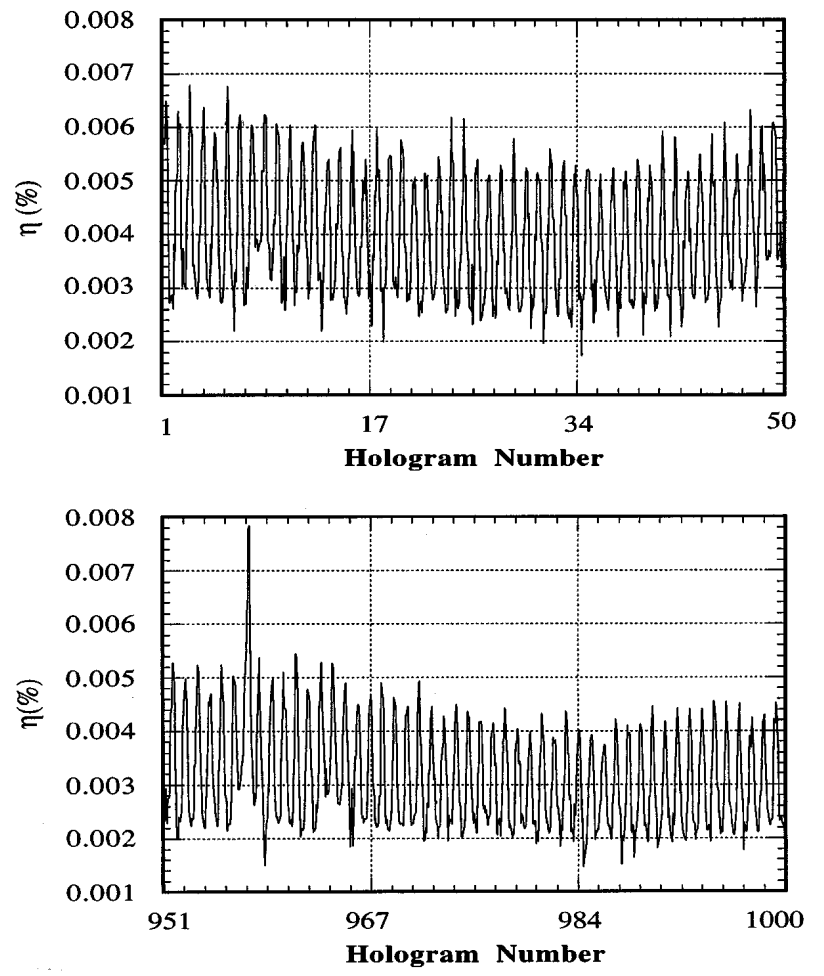

Fig. 3. Diffraction efficiency of 100 multiplexed holograms selected from among 1000.
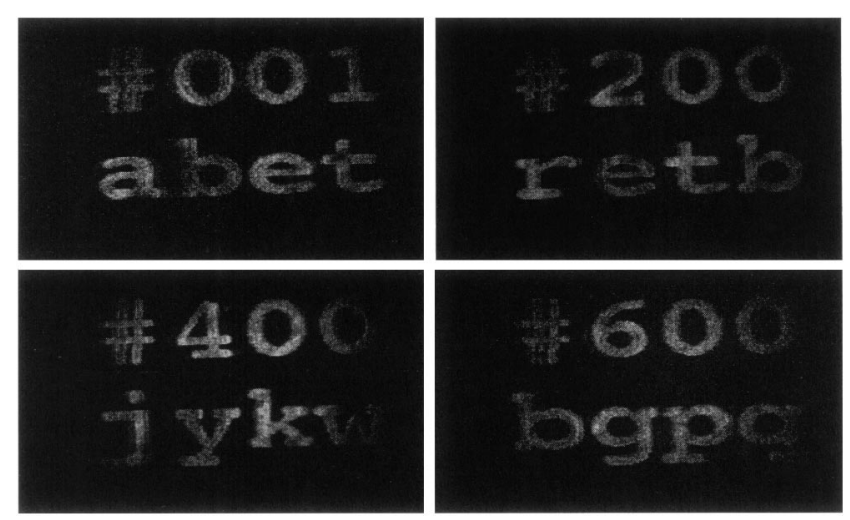

Fig. 4. Samples of reconstructed images from fixedrevealed holograms. The numbers in the pictures are the hologram numbers.

number of exposures in each cycle. By requiring that $A_{m-1}=A_{m}$ for all $m$, we obtain the exposure time schedule:

$$
\begin{aligned}
t_{m-1}= & -\tau_{w} \ln \left(1-\left[1-\exp \left(-\frac{t_{m}}{\tau_{w}}\right)\right] \exp \left(-\frac{t_{m+1}}{\tau_{s}}\right)\right. \\
& \left.\times\left\{\frac{r_{1}+r_{2} \exp \left[S_{m+1}\left(\frac{1}{\tau_{s}}-\frac{1}{\tau_{L}}\right)\right]}{r_{1}+r_{2} \exp \left[S_{m}\left(\frac{1}{\tau_{s}}-\frac{1}{\tau_{L}}\right)\right]}\right\}\right)
\end{aligned}
$$

Mulitplexed recording was performed in five exposure cycles (i.e., one fixing pulse was applied after the 200 holograms of each row were recorded). The depoling pulse and the fixing pulse were both $-1.8 \mathrm{kV} / \mathrm{cm}$ in amplitude and $0.2 \mathrm{~s}$ in duration, and the revealing pulse was $5 \mathrm{kV} / \mathrm{cm}$ in amplitude and $0.5 \mathrm{~s}$ in duration. Figure 3 shows the diffraction efficiency of the first 50 (1 to 50) and the last 50 (951 to 1000) holograms. The average diffraction efficiency of the fixed-revealed holograms was $\sim 0.005 \%$, with a uniformity of $\sim 80 \%$ among different holograms measured by a Bragg-matched extraordinarily polarized beam of $633 \mathrm{~nm}$. Figure 4 shows a sample of images reconstructed from fixed-revealed holograms. A 532-nm wavelength was used to reconstruct the image sample to avoid the partial Bragg-matching effects that occur when the wavelength of reconstruction is different from that of recording. However, some residual distortion exists (the intensity is brighter at the center) because the holograms were recorded by an $o$-polarized beam (to reduce fanning noise during recording) and read out by an $e$-polarized beam (for high diffraction efficiency). Therefore the effective wavelengths are different between recording and reading.

This research was funded in part by the Defense Advanced Research Projects Agency through the PRISM (MDA972094-0008) program.

\section{References}

1. D. L. Steabler, W. J. Burke, W. Phillips, and J. J. Amodei, Appl. Phys. Lett. 26, 182 (1975).

2. F. Micheron and G. Bismuth, Appl. Phys. Lett. 20, 79 (1972); 23, 71 (1973).

3. Y. Qiao, S. Orlov, D. Psaltis, and R. R. Neurgaonkar, Opt. Lett. 18, 1004 (1993).

4. M. Horowitz, A. Bekker, and B. Fischer, Opt. Lett. 22, 1964 (1993).

5. V. Leyva, A. Agranat, and A. Yariv, Opt. Lett. 16, 554 (1991).

6. J. F. Heanue, M. C. Bashaw, A. J. Daiber, R. Snyder, and L. Hesselink, Opt. Lett. 21, 1615 (1996).

7. R. S. Cudney, J. Fousek, M. Zgonik, P. Gunter, M. H. Garrett, and D. Rytz, Phys. Rev. Lett. 72, 3883 (1994).

8. J. Ma, T. Chang, J. Hong, and R. Neurgaonkar, Phys. Rev. Lett. 78, 2960 (1997).

9. H. Lee, X. G. Gu, and D. Psaltis, J. Appl. Phys. 65, 219 (1989).

10. D. Psaltis, D. Brady, and K. Wagner, Appl. Opt. 27, 1752 (1988).

11. G. C. Valley, Appl. Opt. 22, 3160 (1983). 